\title{
CD9 Expression on Lymphatic Vessels in Head and Neck Mucosa
}

Boban M. Erovic, M.D., Csilla Neuchrist, M.D., Sandra Kandutsch, M.D., Markus Woegerbauer, M.S., Johannes Pammer, M.D.

Departments of Otorhinolaryngology, Head and Neck Surgery (BME, CN), and Clinical Pathology (SK, JP), University of Vienna Medical School, Vienna, Austria; and Department of Research and Development, Birkmayer Laboratories (MW), Vienna, Austria

CD9, a member of the transmembrane 4 superfamily, is involved in cell adhesion, migration, and tumor metastasis. Little is known about its vascular expression pattern. In this study, we investigated CD9 expression on endothelial cells in the mucosa of the head and neck and compared it with vascular tumors. Using immunohistochemistry, expression of CD9 was studied in 17 samples of head and neck mucosa and skin (laryngeal mucosa: $n=2$, oral: $n=$ 6, and epidermis: $n=9$ ) and a variety of vascular tumors (lymphangiomas: $\boldsymbol{n}=\mathbf{9}$, juvenile nasopharyngeal angiofibromas: $n=4$, hemangiomas: $n=7$, angiosarcomas: $n=5$, and Kaposi's sarcomas: $n=$ 7) and compared with the expression of CD34 and PAL-E (blood vessel markers) and the lymphatic marker podoplanin. Regular lymphatic endothelium and lymphangiomas were strongly positive for CD9 and podoplanin but were mostly negative for PAL-E and CD34. By contrast, blood vessel endothelium and hemangiomas were strongly positive for PAL-E and CD34 but were mostly negative for CD9 and podoplanin. Weak to moderate CD9 reactivity was also observed on EC of juvenile nasopharyngeal angiofibromas, angiosarcomas, and Kaposi's sarcomas. Expression of CD9 by lymphatic EC was confirmed by reverse-transcriptase PCR and Western blot analyses. CD9 may be useful as a marker for lymphatic EC. It could promote the adherence of inflammatory and tumor cells to lymphatic EC and participate in the growth and maintenance of the lymphatic capillary net.

\footnotetext{
Copyright (C) 2003 by The United States and Canadian Academy of Pathology, Inc.

VOL. 16, NO. 10, P. 1028, 2003 Printed in the U.S.A.

Date of acceptance: June 20, 2003.

Supported by the Fonds zur Foerderung der Wissenschaftlichen Forschung SFB 05-Contract 007. TX dk

Address reprint requests to: Johannes Pammer, M.D., Department of Clinical Pathology, University of Vienna Medical School, Allgemeines Krankenhaus, Währinger Gürtel 18-20, A-1097 Wien, Austria; fax: +43-140400-3690; e-mail: johannes.pammer@akh-wien.ac.at.

DOI: 10.1097/01.MP.0000089777.58000.B2
}

KEY WORDS: CD9, Head and neck mucosa, Lymphatic endothelium, Vessels.

Mod Pathol 2003;16(10):1028-1034

CD9, also known as motility-related-protein-1 (MRP-1), is a member of the transmembrane 4 superfamily (1). It is widely expressed in healthy tissue (2) as well as in various tumors $(3,4)$, and it is involved in cell motility by virtue of its interaction with VLA integrins (5). CD9 influences proliferation in a variety of cell types (6), and its ectopic overexpression can slow down cell growth by elongating the doubling time of DNA replication (7).

Anti-CD9 antibodies have also been reported to inhibit endothelial cell migration, enhance their proliferation, and inhibit endothelial cell apoptosis $(8,9)$. In addition, CD9 expression on endothelial cell has been reported to promote transmigration of tumor cells through endothelial cell layers (10). Therefore, endothelial CD9 may play a critical role in wound healing and angiogenesis, and EC survival may be pivotal to metastasis of solid tumors.

We recently detected a subset of vessels that were intensely positive for CD9 in head and neck mucosa and head and neck squamous cell carcinomas (26). These vessels were mainly located subepithelially in normal mucosa and, to a lesser extent, also in peritumoral stroma. Further immunohistochemical typing in serial sections with podoplanin revealed strong evidence that these CD9-positive vessels were of lymphatic origin.

In this study, we investigated the hypothesis that strong CD9 expression was specific for lymphatic endothelium. Vessels of head and neck mucosa were stained for CD9, podoplanin (a specific marker for lymphatic endothelial cells (11), and PAL-E and CD34 (both markers for EC of blood vessels) (12), and their expression pattern was compared with that of vascular tumors. RT-PCR and Western blot analyses were employed to analyze CD9 mRNA and protein expression in cultivated lymphatic and blood endothelial cells. 


\section{MATERIALS AND METHODS}

\section{Tissue Samples}

Formalin-fixed, paraffin-embedded tissue blocks of 17 specimens of oral mucosa and skin of the head and neck (oral mucosa, $n=6$; larynx, $n=2$; epidermis, $n=9$ ), 12 specimens of internal organs (kidney: $n=2$, lung: $n=2$, duodenum: $n=3$, and colon: $n=5$ ), and a variety of vascular tumors (9 lymphangiomas, 7 hemangiomas, 4 juvenile nasopharyngeal angiofibromas, 5 angiosarcomas, and 7 Kaposi's sarcomas as well as 2 peripheral giant cell granulomas of the jaw) were obtained from the files of the Institute of Clinical Pathology at the University of Vienna. Frozen sections of lung $(n=1)$, stomach $(n=1)$, and colon $(n=4)$ were obtained from surgical preparations that were snap frozen in OCT embedding media and stored at $-80^{\circ} \mathrm{C}$.

\section{Immunohistochemistry}

Samples were immunostained for CD9 using monoclonal antibody 72F6 (Novocastra Laboratories, Newcastle upon Tyne, UK). Antigen retrieval was performed by boiling sections in a microwave oven in 1 mm EDTA (pH 8.0) buffer for 20 minutes. Primary antibody was applied at 1:500 dilution overnight, followed by a biotinylated $\alpha$-mouse antibody (1:100; Vector Laboratories, Burlingame, CA) and alkaline phosphatase conjugated streptavidin complex (1:250; DAKO, Glostrup, Denmark). The enzyme reaction was developed with an alkaline phosphatase substrate kit (1:250; Vector Laboratories). Negative controls were carried out on consecutive tissue sections using isotype-matched control reagents $\left(\mathrm{IgG}_{1}\right.$, Coulter, Hialeah, FL, 1:250, and $\mathrm{IgG}_{1}$, PharMingen, San Diego, CA, 1:250).

Serial sections of some tissues were treated with a rabbit serum against podoplanin (kindly provided by Prof. Dr. Sylvana Breiteneder-Geleff and Prof. Dr. Dontscho Kerjaschki; $0.0025 \mu \mathrm{g} / \mathrm{mL}$ ) (11), a rabbit control serum (diluted 1:200) or a monoclonal antibody against CD34 (clone Qbend/10, $2 \mu \mathrm{g} / \mathrm{mL}$, 1:500; Serotec, Oxford, England), processed by an indirect immunohistochemical method using a standard avidin-biotin (CD34) and streptavidinbiotin (podoplanin) staining technique and developed with diaminobenzidine tetrahydrochloride dehydrate (Abbott, Wiesbaden, Germany).

For PAL-E staining, 4- $\mu \mathrm{m}$-thick frozen sections were fixed in ice-cold acetone and in 4\% PFA and incubated with TBS-10\% human serum-5\% BSA to block nonspecific binding. Monoclonal antibody $\alpha$-PAL-E (1:20; Harlan-Sera Lab, Loughborough, England) was incubated for 1 hour at room temperature. Slides were subsequently exposed to Texas red (Alexa-fluor 594) $\mathrm{f}(\mathrm{ab})_{2}$ fragments of goat $\alpha$-mouse IgG (1:1000; Molecular Probes, Eugene,
OR) for 1 hour at room temperature, and after extensive washing, they were incubated with $\alpha$-FITClabeled CD9 monoclonal antibody (1:50; Ancell, Bayport, MN) for 1 hour at room temperature. Staining was visualized with a confocal Laser Scanning Microscope (CLSM 410, Zeiss; original magnification, $400 \times$ ).

Reactivity for CD9, podoplanin, and CD34 was determined as low (reactivity in $0-10 \%$ or $10-40 \%$ of all vessels), moderate $(40-60 \%$ or $60-80 \%)$, or high $(80-100 \%)$.

\section{Cell Culture}

Cell culture was performed as described previously (12). Briefly, microvascular endothelial cells adjusted to $10^{7}$ cells $/ \mathrm{mL}$ in endothelial cells growth medium MV (Clonetics, Verviers, Belgium) were exposed simultaneously to rabbit-anti-podoplanin serum (final concentration: 1:100), anti-CD34-PE (Becton Dickinson, Franklin Lakes, NJ), and antiCD45-RPE-Cy5 (Serotec, Oxford, UK) for $45 \mathrm{~min}$ utes on ice. Goat anti-mouse f(ab') $)_{2}$ FITC $(10 \mu \mathrm{g} /$ mL, Jackson ImmunoResearch Laboratories, West Grove, PA) was added, and cells were subjected to flow cytometric analyses.

CD34 ${ }^{+} \mathrm{CD} 45^{-}$endothelial cells were sorted into podoplanin $^{-}$or podoplanin ${ }^{+}$populations on a FACScan (Becton Dickinson). On fibronectin (10 $\mu \mathrm{g} / \mathrm{mL}$ )-coated 96-well flat-bottom plates (Becton Dickinson), $0.5 \times 10^{6}$ cells $/ \mathrm{mL}$ were cultured in endothelial cell growth medium MV (Clonetics, Verviers, Belgium).

\section{RT-PCR}

Total RNA from FACS-isolated microvascular endothelial cells was isolated as described previously (13). Single-stranded cDNA was obtained by reverse transcription of $1 \mu \mathrm{g}$ of total RNA according to manufacturer's instructions (First strand cDNA Kit, 5 Prime-3 Prime Inc., Boulder, CO). Reaction mixtures were incubated at $42^{\circ} \mathrm{C}$ for 1 hour, and excess enzyme was inactivated at $75^{\circ} \mathrm{C}$ for $10 \mathrm{~min}$. The CD9 sequence (EMBL/Genbank accession number X60111.1) was used as template sense and antisense primers: 5'-TGCATCTGTATCCAGCGCCA-3'and 5'CTCAGGGATGTAAGCTGACT-3' (14). Amplification products were separated on a $1.5 \%$ agarose gel and visualized under ultraviolet light in the presence of ethidium bromide.

\section{Western Blot Analyses}

To confirm the specificity of CD9 antibody, Western blot analyses were performed using proteins extracted from isolated lymphatic endothelial cells and blood endothelial cells, which were sorted on a FACScan. SDS-polyacrylamide gel electrophoresis 

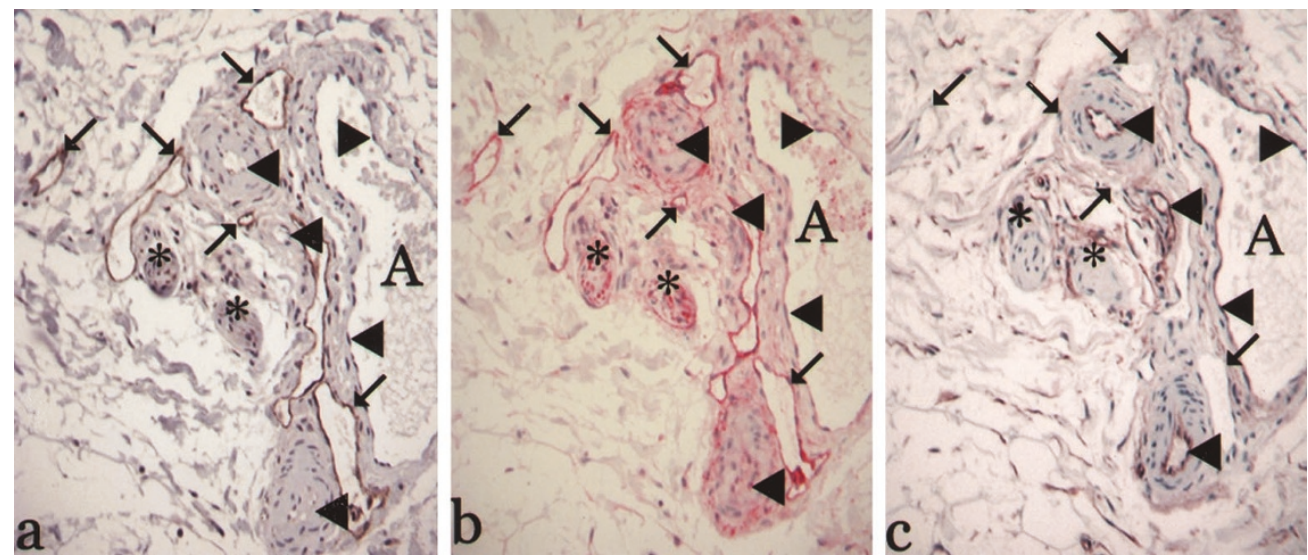

FIGURE 1. Expression of CD9 on lymphatic and blood vascular endothelial cells. Flat-lined vessels (small arrows) are strongly positive for podoplanin (A, small arrows) and CD9 (B) but negative for CD34 (C). By contrast, blood vessels are distinctly positive for CD34 (C, large arrowheads), only weakly positive for CD9 and podoplanin (B, A). CD9-positive nerves are marked with asterisks (magnification, 200×).

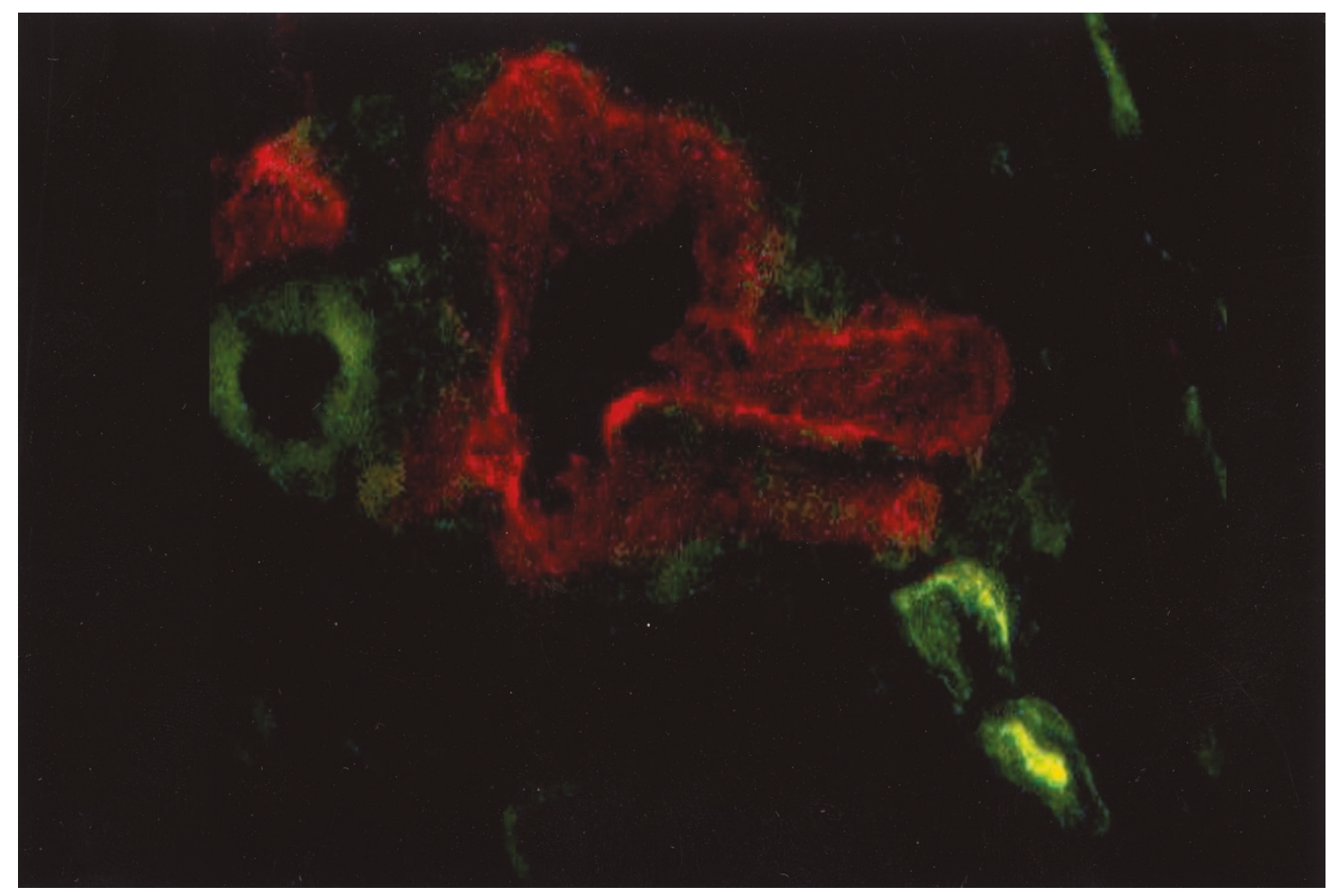

FIGURE 2. CD9 IR on lymphatic endothelial cells in head and neck mucosa. Frozen sections of laryngeal mucosa immunostained with $\alpha$-CD9FITC (green) and $\alpha$-PAL-E (red). Red and green spots indicate endothelial cell lining on blood capillaries and two lymphatic capillaries, respectively (magnification, $400 \times$ ).

was performed with a 3.5 to $15 \%$ gradient gel using a standardized system (Bio-Rad Laboratories, Richmond, CA). The electrophoresed proteins were transferred overnight to a nitrocellulose membrane (Schleicher \& Schuell, Dassel, Germany) and subjected to Western blot analyses with the monoclonal antibody CD9 (diluted 1:10). Immunoreactivity for CD9 protein was visualized with an ECL kit (Amersham, Arlington Heights, IL), and chemoluminescence analyses of the CD9 band were done by Lumi Imager (Boehringer Mannheim, Germany).

\section{RESULTS}

CD9, Podoplanin, PAL-E, and CD34 Expression in the Mucosa and Skin of the Head and Neck

Eighty to $100 \%$ of mucosal and $40-60 \%$ of dermal lymphatic vessels, recognized by flat-lined endothelium, the lack of intravascular erythrocytes, as well as a smooth muscle cell layer, expressed CD9 distinctly (Fig. 1B). These CD9-positive vessels were mainly located subepithelially. In serial sections, endothelial cells of these vessels were also 


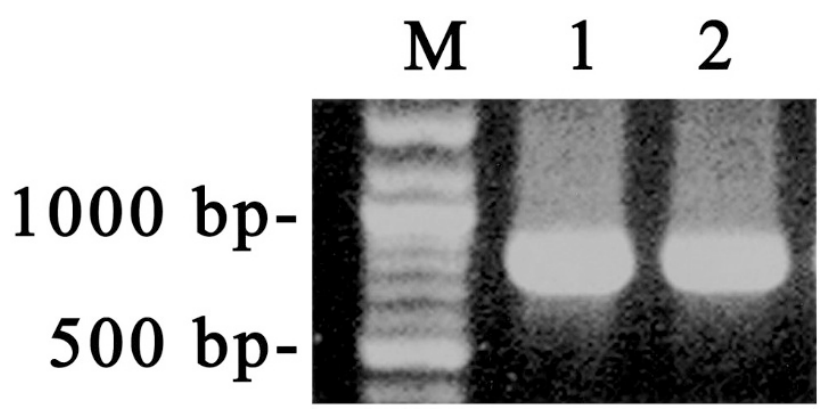

FIGURE 3. Expression pattern of CD9 mRNA is shown by RT-PCR in cultured lymphatic and blood endothelial cells. In both cell lines (Lanes 2 and 3), a distinct band of approximately $800 \mathrm{bp}$ is detected. In the first lane, $\mathrm{H}_{2} \mathrm{O}$ was used as negative control ( $\mathrm{m}=$ marker, $1=$ lymphatic, and 2 = blood endothelial cells).

positive for podoplanin (Fig. 1A). In addition, a smaller subset $(10-20 \%)$ of blood vessels both of the mucosa and the skin had a weakly CD9positive endothelial cell layer (data not shown). Whereas lymphatics distinctly positive for CD9 were mostly CD34 negative (Fig. 1C), blood vessel endothelia were positive for CD34 in serial sections. This expression pattern was also found in kidney, lung, and gastrointestinal tissue, as well as in small vessels around two peripheral giant cell granulomas, indicating that it was typical for vessels of all organs and also was preserved in inflammatory reactions. Nerves and squamous epithelia (2) served as an internal positive control for CD9-staining.

For further confirmation of the relative specificity of CD9 for lymphatic endothelia, we stained cryosections of mucosal tissue with both FITC-labeled CD9 and PAL-E monoclonal antibodies. As expected from the above staining results, we found that endothelial cells expressed exclusively either CD9 or PAL-E (Fig. 2).

To confirm the potential of lymphatic endothelial cells to express CD9, RT-PCR was performed using mRNA of blood and lymphatic endothelial cells that were sorted on a FACScan. RT-PCR revealed a

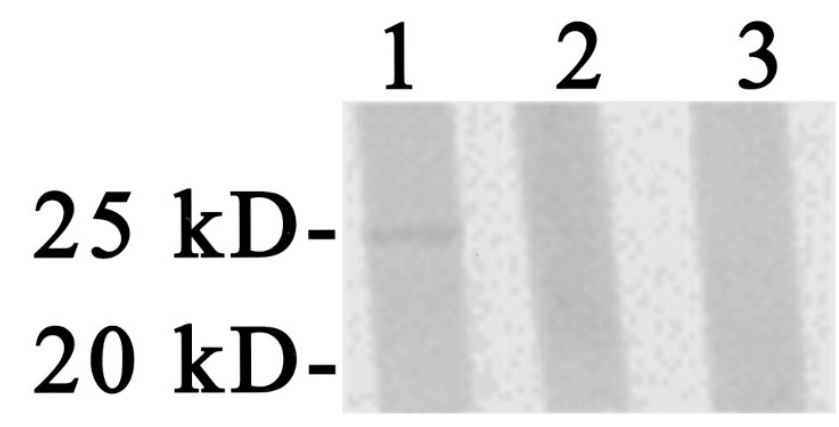

FIGURE 4. CD9 protein expression by western blot analyses in cultured lymphatic and blood endothelial cells. At approximately $25 \mathrm{kD}$ a single band is only observed in lymphatic endothelial cells (Lane 1). No protein expression was observed in blood endothelial cells (Lane 2) and the negative control (Lane 3).
Table 1. CD9 Expression on Lymphatic and Blood Endothelial Cells of Vascular Tumors

\begin{tabular}{|c|c|c|c|}
\hline Number & \multicolumn{3}{|c|}{ Lymphangiomas } \\
\hline & CD34 & CD9 & Podoplanin \\
\hline 1 & 0 & $40-60$ & nd \\
\hline 2 & $0-10$ & $60-80$ & nd \\
\hline 3 & $0-10$ & $60-80$ & nd \\
\hline 4 & 0 & $60-80$ & $80-100$ \\
\hline 5 & $0-10$ & $80-100$ & nd \\
\hline 6 & $0-10$ & $80-100$ & $80-100$ \\
\hline 7 & $0-10$ & $80-100$ & nd \\
\hline 8 & 0 & $80-100$ & $80-100$ \\
\hline 9 & 0 & $80-100$ & $80-100$ \\
\hline \multicolumn{4}{|c|}{ Hemangiomas } \\
\hline 1 & nd & $0-10$ & nd \\
\hline 2 & nd & 0 & nd \\
\hline 3 & nd & $0-10$ & nd \\
\hline 4 & nd & $0-10$ & nd \\
\hline 5 & $80-100$ & $0-10$ & nd \\
\hline 6 & $80-100$ & $0-10$ & $0-10$ \\
\hline 7 & nd & $0-10$ & nd \\
\hline \multicolumn{4}{|c|}{ Angiofibromas } \\
\hline 1 & nd & 0 & nd \\
\hline 2 & $80-100$ & $40-60$ & $40-60$ \\
\hline 3 & nd & $0-10$ & nd \\
\hline 4 & nd & 0 & nd \\
\hline \multicolumn{4}{|c|}{ Angiosarcomas } \\
\hline 1 & 0 & 0 & 0 \\
\hline 2 & nd & $60-80$ & nd \\
\hline 3 & nd & $60-80$ & nd \\
\hline 4 & $80-100$ & 0 & nd \\
\hline 5 & $80-100$ & 0 & nd \\
\hline \multicolumn{4}{|c|}{ Kaposi's sarcomas } \\
\hline 1 & $40-60$ & $60-80$ & $60-80$ \\
\hline 2 & $20-40$ & $60-80$ & $60-80$ \\
\hline 3 & $0-10$ & $60-80$ & $60-80$ \\
\hline 4 & $80-100$ & $20-40$ & $20-40$ \\
\hline 5 & $40-60$ & $80-100$ & $80-100$ \\
\hline 6 & $80-100$ & $80-100$ & $80-100$ \\
\hline 7 & $80-100$ & $60-80$ & $80-100$ \\
\hline
\end{tabular}

nd $=$ not done.

strong single band of approximately $800 \mathrm{bp}$ in both cell types (Fig. 3).

The expression pattern of CD9 protein in these blood and lymphatic endothelial cells was further analyzed by Western blotting. A strong single CD9 band of approximately $25 \mathrm{kDa}$ was found in isolated lymphatic endothelial cells. Blood endothelial cells and the negative control did not show any protein bands (Fig. 4).

\section{Coexpression of CD9 and Podoplanin in Benign Vascular Tumors (Table 1)}

Immunohistochemical analyses demonstrated distinct CD9 expression in most EC of nine lymphangiomas (Table 1, Fig. 5B). The lymphatic differentiation of four of these tumors was demonstrated by staining for podoplanin, which labeled $80-100 \%$ of their endothelial cells (Fig. 5A). Weak labeling for CD34 was observed on minor parts of these tumors (0-10\%) (Fig. 5C, arrows).

By contrast, only a minor part $(0-10 \%)$ of capillaries in hemangiomas expressed CD9 by immunohistochemistry (Fig. 5E, arrows). These vessels 


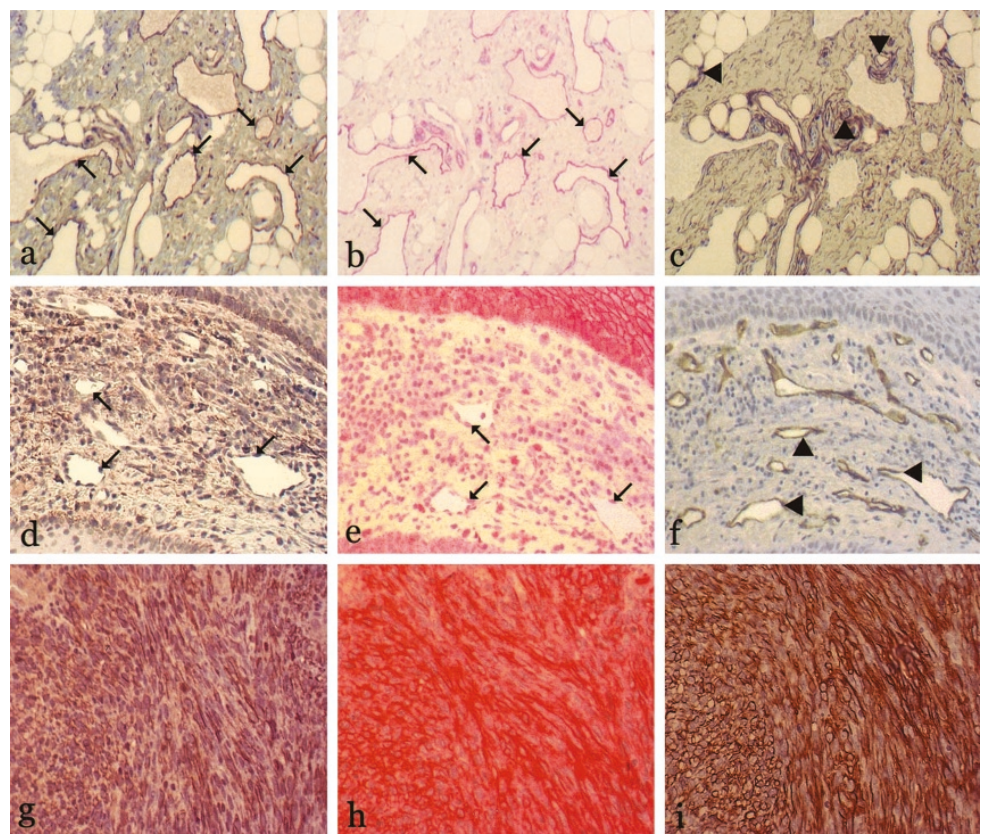

FIGURE 5. Expression of podoplanin, CD9, and CD34 in vascular tumors. Small flat-lined vessels of this lymphangioma were strongly positive for podoplanin (A, arrows) and for CD9 (B, arrows) but negative for CD34 (C). Small capillaries that were negative for CD9 stained intensely for CD34 (C, arrowheads, magnification $100 \times$ ). Small vessels of a capillary hemangioma showed nearly no podoplanin (D, arrows) and CD9 reactivity (E, arrows) but were CD34 positive (F, arrowheads, magnification $200 \times$ ). Tumor cells of Kaposi's sarcomas demonstrate a high immunoreactivity for all three antibodies (G-I, magnification $200 \times$ ).

were distinctly CD34 positive (80-100\%; Fig. 5F), but podoplanin negative (Fig. 5D). Furthermore, most (80-100\%) CD34-positive tumor vessels in three of four juvenile nasopharyngeal angiofibromas were negative for CD9 (Table 1). In one angiofibroma, $40-60 \%$ of endothelial cells of the tumor vessels expressed both CD9 and podoplanin.

\section{CD9, Podoplanin, and CD34 Expression in Malignant Vascular Tumors (Table 1)}

In only two of five angiosarcomas, $60-80 \%$ of tumor cells expressed CD9 weakly, whereas in three of five specimens, no CD9 expression could be detected (Table 1). By contrast, the majority of tumor cells showed distinctly and focally strong CD9 expression in Kaposi's sarcomas (Fig. 5H). Most CD9positive tumor cells in Kaposi's sarcomas were also podoplanin positive (Fig. 5G). In addition, Kaposi's sarcoma tumor cells expressed CD34 (Fig. 5I), as described previously (15).

Similar to healthy mucosa and skin, most lymphatic vessels adjacent to benign and malignant tumors expressed CD9 and podoplanin, whereas blood vessels were mainly negative for CD9 but positive for CD34. These findings are generally summarized in Table 1.

\section{DISCUSSION}

This study describes for the first time the detailed expression pattern of CD9 on endothelial cells and vascular tumors. We show that CD9 expression as detected by monoclonal antibody clone $72 \mathrm{~F} 6$ is mainly expressed on lymphatic endothelial cells, whereas blood vessel endothelial cells are either negative or only weakly positive for CD9 by immunohistochemistry. In addition, these data are substantiated by RT-PCR and Western blot analyses of FACS-sorted endothelial cells. Also human umbilical vein endothelial cells expressed mRNA CD9; however, mRNA was not quantified by additional experiments.

The expression of CD9 by endothelial cells has been demonstrated previously by FACS analyses (8), but there are only few studies on the distribution of this antigen in situ. Aortic and coronary endothelia of normal vessels have been reported to be negative for CD9 by immunohistochemistry (6). In addition, glomerular endothelial cells were also found to lack CD9 expression (16). By contrast, a comprehensive analysis demonstrated strong CD9 expression on vascular endothelium of arteries and veins and to a lesser degree on capillaries in a variety of organs (2). The reason for these differences is not clear-they may be due to the glycosylation state of CD9 (17) or to its binding to other proteins, such as integrins or other tetraspanins (18), resulting in epitope masking depending on the antibody used.

Our demonstration that little CD9 is expressed on blood vessel endothelia is therefore in accordance with a variety of earlier reports. In addition, the expression pattern of CD9 on epithelial cells and 
nerves in our study was identical to those described by other investigators (2), confirming the validity of our observations.

However, the expression of CD9 on lymphatic endothelial cells was not addressed in these other studies. By Western blotting and staining serial sections for CD9 and specific markers for blood and lymphatic endothelial cells, that is, $\operatorname{CD} 34(11,12)$, PAL-E (19), and podoplanin (11), we showed that CD9 is a marker that is possibly useful in identifying lymphatic endothelial cells similar to more specific markers such as VEGFR3/flt-4 (a receptor of VEGF-C (20), LYVE-1 (a hyaluron receptor) (21), D6 (a $\beta$-chemokine receptor) (22), and podoplanin (whose function is still unknown) (11). The expression pattern of distinct CD9 reactivity of lymphatic, but not blood vessel endothelial cells, was also preserved on endothelial cells of benign vascular tumors. The expression of CD9 on Kaposi sarcoma spindle cells is in accordance with their podoplanin expression and may thus be regarded as a further indication of their lymphatic origin (23).

A potential role for tetraspanins in adhesion has been shown in a variety of studies (24). Strong expression of CD9 may enhance the adherence of neutrophils to lymphatics (25) or promote transmigration of tumor cells through the lymphatic endothelial cells layer (10) and could therefore play a role in the immune response, inflammation, and tumor metastasis.

Furthermore, CD9 has been shown to be involved in endothelial cells migration and growth (8) and may thus participate in the growth and maintenance of the lymphatic capillary net.

In summary, we have described that intense CD9 expression can be useful as a new marker for lymphatic endothelial cells. This strong expression may be mandatory for growth, maintenance and pathophysiology of lymphatic vessels. The relevance of this finding needs further investigation and may lead to discoveries of new aspects in the biology and pathological processes of lymphatic vessels.

Acknowledgments: The authors thank Prof. D. Kerjaschki for substantial support, Marianne Peterlik, Ingrid Raab, and Romana Kalt for their excellent technical assistance; Georg E. Steiner and Dietmar Thurnher for assistance with the graphical work; and Heidemarie Rossiter and Michael B. Fischer for proofreading and valuable discussion.

\section{REFERENCES}

1. Boucheix C, Rubinstein E. Tetraspanins. Cell Mol Life Sci 2001;58:1189-205.

2. Sincock PM, Mayrhofer G, Ashman LK. Localization of the transmembrane 4 superfamily (TM4SF) member PETA-3
(CD151) in normal human tissues: comparison with CD9, CD63, and alpha5betal integrin. J Histochem Cytochem 1997;45:515-25.

3. Mori M, Mimori K, Shiraishi T, Haraguchi M, Ueo H, Barnard GF, et al. Motility-Related-Protein-1 (MRP-1/CD9) expression in colon cancer. Clin Cancer Res 1998;4:1507-10.

4. Miyake M, Adachi M, Huang C, Higashiyama M, Kodama K, Taki T. A novel molecular staging protocol for non-small cell lung cancer. Oncogene 1999;18:2397-404.

5. Rubinstein E, Le Naour F, Lagaudriere-Gesbert C, Bilard M, Conjeaud H, Boucheix C. CD9, CD63, CD81 and CD82 are components of a surface tetraspan network connected to HLA-DR and VLA integrins. Eur J Immunol 1996;26: 2657-65.

6. Nishida M, Miyagawa J, Yamashita S, Higashiyama S, Nakata A, Ouchi N, et al. Localization of CD9, an enhancer protein for proheparin-binding epidermal growth factor-like growth factor, in human atherosclerotic plaques: possible involvement of juxtacrine growth mechanism on smooth muscle cell proliferation. Arterioscler Thromb Vasc Biol 2000;20:1236-43.

7. Ikeyama S, Koyama M, Yamaoko M, Sasada R, Miyake M. Suppression of cell motility and metastasis by transfection with human motility related protein-1 (MRP-1/CD9) DNA. J Exp Med 1993;177:1231-7.

8. Klein-Soyer C, Azorsa DO, Cazenave JP, Lanza F. CD9 participates in endothelial cell migration during in vitro wound repair. Arterioscler Thromb Vasc Biol 2000;20:360-9.

9. Araki S, Masuda S, Maeda H, Ying MJ, Hayashi H. Involvement of specific integrins in apoptosis induced by vascular apoptosis-induced protein 1. Toxicon 2002;40:535-42.

10. Longo N, Yanez-Mo M, Mittelbrunn M, de la Rosa G, Munoz ML, Sanchez-Madrid F, et al. Regulatory role of CD9 in tumor-endothelial cell interaction during transendothelial invasion of melanoma cells. Blood 2001;98:3717-26.

11. Breiteneder-Geleff S, Soleiman A, Kowalski H, Horvat R, Aman G, Kriehuber E, et al. Angiosarcomas express mixed endothelial phenotypes of blood and lymphatic capillaries. Podoplanin as a specific marker for lymphatic endothelium. Am J Pathol 1999;154:385-94.

12. Kriehuber E, Breiteneder-Geleff S, Groeger M, Soleiman A, Schoppmann SF, Stingl G, et al. Isolation and characterization of dermal lymphatic and blood endothelial cells reveal stable and functionally specialized cell lineages. J Exp Med 2001;194:797-808.

13. Adachi M, Taki T, Ieki Y, Huang C, Higashiyama M, Miyake M. Correlation of KAI1/CD82 gene expression with good prognosis in patients with non-small cell lung cancer. Cancer Res 1996;56:1751-5.

14. Miyake M, Nakano K, Ieki Y, Adachi M, Huang C, Itoi S, et al. Motility related protein 1 (MRP-1/CD9) expression: inverse correlation with metastases in breast cancer. Cancer Res 1995;55:4127-31.

15. Nickoloff BJ. The human progenitor cell antigen (CD34) is localized on endothelial cells, dermal dendritic cells, and perifollicular cells in formalin-fixed normal skin, and on proliferating endothelial cells and stromal spindle-shaped cells in Kaposi's sarcoma. Arch Dermatol 1999;127: 523-9.

16. Nakamura Y, Handa K, Iwamoto R, Tsukamoto T, Takahasi M, Mekada E. Immunohistochemical distribution of CD9, heparin binding epidermal growth factor-like growth factor, and integrin alpha3betal in normal human tissues. J Histochem Cytochem 2001;49:439-44.

17. Ono M, Handa K, Withers DA, Hakomori S. Motility inhibition and apoptosis are induced by metastasis-suppressing gene product CD82 and its analogue CD9, with concurrent glycosylation. Cancer Res 1999;59:2335-9. 
18. Hadjiargyrou M, Kaprielian Z, Kato N, Patterson PH. Association of the tetraspan protein CD9 with integrins on the surface of S-16 Schwann cells. J Neurochem 1996;67:2505-13.

19. Wilting J, Papoutsi M, Christ B, Nicolaides KH, von Kaisenberg CS, Borges J, et al. The transcription factor Proxl is a marker for lymphatic endothelial cells in normal and diseased human tissues. FASEB J 2002;16:1271-3.

20. Lymboussaki A, Partanen TA, Olofsson B, Thomas-Crussells $\mathrm{J}$, Fletcher CD, de Waal RM, et al. Expression of the vascular endothelial growth factor $C$ receptor VEGFR-3 in lymphatic endothelium of the skin and in vascular tumors. Am J Pathol 1998;153:395-403.

21. Banerji S, Ni J, Wang SX, Clasper S, Su J, Tammi R, et al. LYVE-1, a new homologue of the CD44 glycoprotein, is a lymph-specific receptor for hyaluronan. J Cell Biol 1999; 144:789-801.

22. Nibbs RJB, Kriehuber E, Ponath PD, Parent D, Qin S, Camp- bell JDM, et al. The $\beta$-chemokine receptor D6 is expressed by lymphatic endothelium and a subset of vascular tumors. Am J Pathol 2001;158:867-77.

23. Weninger W, Partanen TA, Breiteneder-Geleff S, Mayer C, Kowalski H, Mildner M, et al. Expression of vascular endothelial growth factor receptor-3 and podoplanin suggests a lymphatic endothelial cell origin of Kaposi's sarcoma tumor cells. Lab Invest 1999;79:243-51.

24. Levy S, Todd SC, Maecker HT. CD81 (TAPA-1): a molecule involved in signal transduction and cell adhesion in the immune system [review]. Annu Rev Immunol 1998;16:89109.

25. Forsyth KD. Anti-CD9 antibodies augment neutrophil adherence to endothelium. Immunology 1991;72:292-6.

26. Erovic BM, Pammer J, Hollemann D, et al. Motility-related protein-1/CD9 expression in head and neck squamous cell carcinoma. Head Neck 2003;25:848-57. 